\title{
Study of "Low-Frequency" Seismic Events Sources in the Mines of the Verkhnekamskoye Potash Deposit
}

\author{
D.A. Malovichko Mining Institute, Ural Branch, Russian Academy of Sciences, Russia
}

Seismic networks operated in the mines of the Verkhnekamskoye potash deposit routinely register seismic events associated with mining. Intensive low-frequency $(0.5-2 \mathrm{~Hz})$ Rayleigh waves occur on the waveforms of some events (so-called "low-frequency" events). Classical seismological source model in the form of expanding shear crack does not reproduce waveforms of the "low-frequency" events: high-frequency body waves dominate on synthetic waveforms for double-couple point sources with reliable parameters. Dynamical processes observed in potash mines (pillar burst, rockfall, dynamical floor failure) have been analyzed as potential sources of the "low-frequency" seismic events. The equivalent point sources have been determined for these processes. Synthetic waveforms for constructed point sources have been calculated and compared with seismograms of the "low-frequency" events. It has been found that only rockfall could generate intensive enough Rayleigh type waves. Rockfalls are expected to be the most probable source of the "low-frequency" seismic events in the Verkhnekamskoye potash deposit.

\section{INTRODUCTION}

\subsection{Seismological Monitoring at the Verkhnekamskoye Potash Deposit}

The Verkhnekamskoye potash deposit is situated in the Western Urals in the Perm region (Russia). Six mines operate there from one to three productive horizons (in sylvinite and carnallite zones) lying at the depth from 140 to $420 \mathrm{~m}$ below surface (Figure 1). Room-and-pillar system is used in the mines (Mining in the Verknekamskoye potash deposit methodical manual, 1992). The parameters of the rooms: length 150-190 m, width 3-15 m, height up to $10 \mathrm{~m}$. Width of the pillars is $3-18 \mathrm{~m}$.

Seismological observations in the deposit have been started in 1995. The observations are performed using arrays of vertical velocimeters SM3-KV (having flat amplitudefrequency characteristic in the range $0.5-30 \mathrm{~Hz}$ ) installed underground in mine openings. Signals from the velocimeters are transmitted via telemetry systems to ground data acquisition modules.

Seismic events associated with mining are registered routinely. Waveforms of the events are generally represented by the packets of compressional $(\mathrm{P})$ and more intensive shear (S) waves. The frequency range of these waves is $8-20 \mathrm{~Hz}$.
The conventional processing of seismological data includes:

- discrimination of seismic signals and picking up P and $\mathrm{S}$ waves arrivals;

- estimation of the position of the source, the time of the source process initiation and radiated seismic energy for every signal;

- identification of the types of the signals (mine blasts, mine-induced events).

Catalogs of mine-induced events are used in the analysis of seismic regime of the mines.

\subsection{The "Low-Frequency" Seismic Events}

Waveforms of some events are complicated by intensive low-frequencywavetrains(Figure2a).Thenumericalmodeling of seismic waves propagation in the Verknekamskoye deposit medium model (Malovichko and Baranov, 2001) has given an opportunity to interpret those low-frequency waves as Rayleigh ones.

Events with intensive Raileigh waves have symbollicaly been called as "low-frequency". Waveforms of the "low-frequency" events have got some characteristic features in frequency domain (Figure $2 b$ ): there is a flat region in

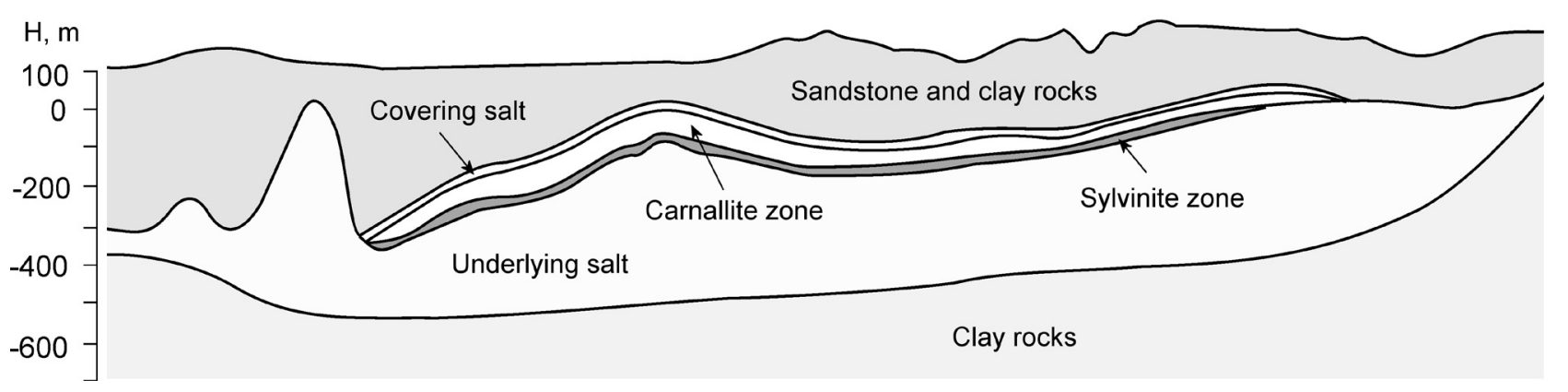


a

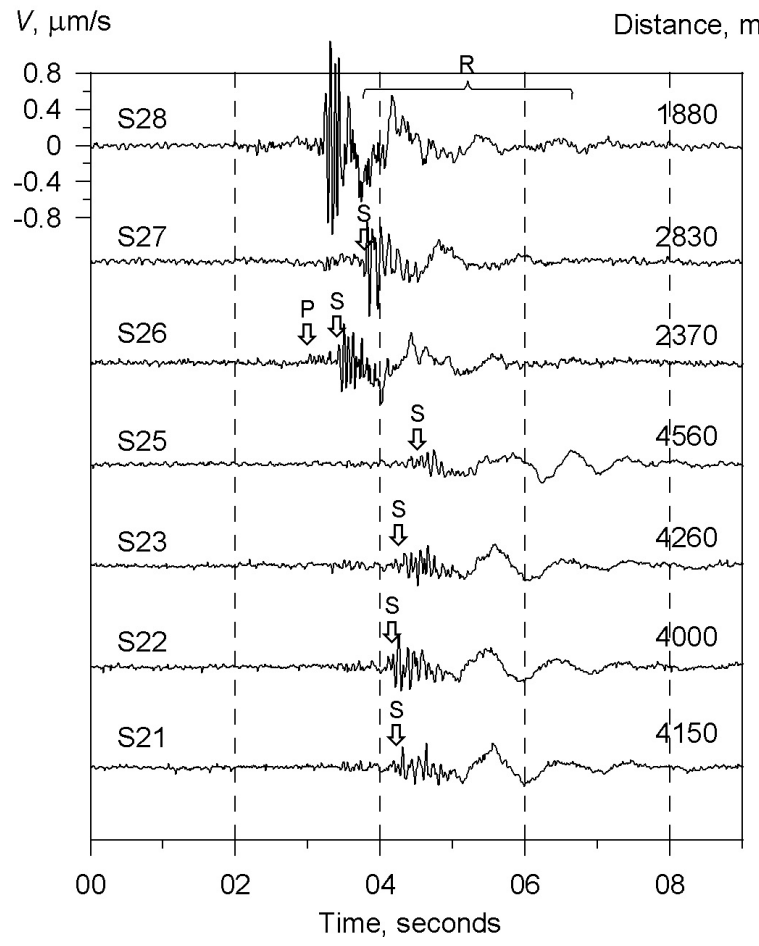

b

$V,(\mu \mathrm{m} / \mathrm{s}) / \mathrm{Hz}$

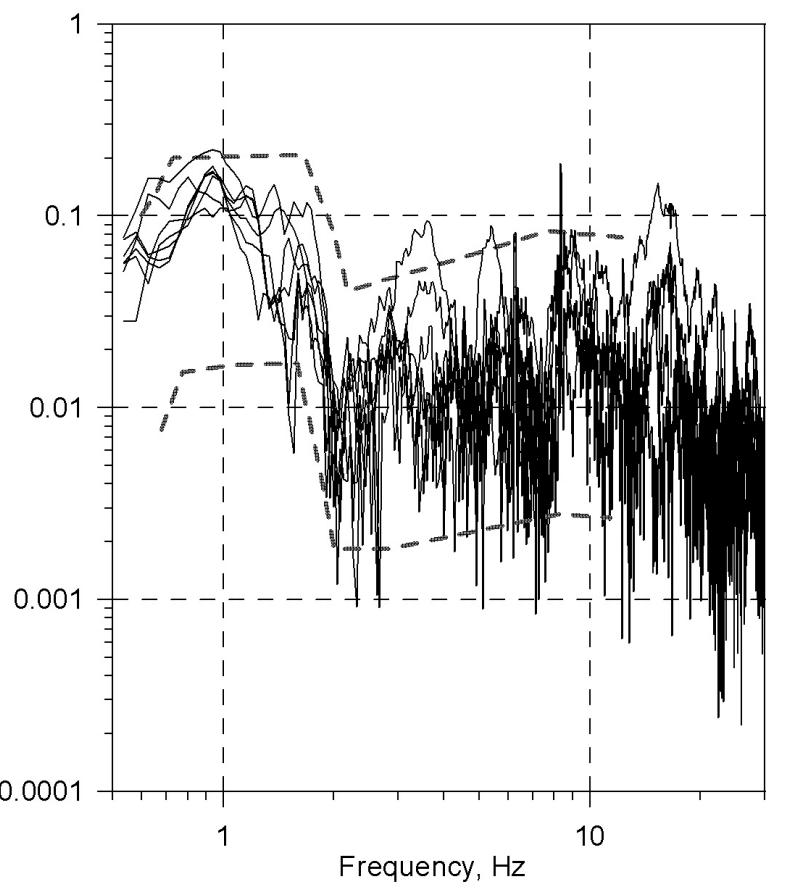

FIG. 2 Waveforms of the "low-frequency" seismic event (a) and their amplitude spectrums (b)

the range $0.5-2 \mathrm{~Hz}$ with spectral amplitudes $0.02-0.2$ $(\mu \mathrm{m} / \mathrm{s}) / \mathrm{Hz}$, an essential falloff is noted on higher frequencies and there is an enhancement of spectral amplitudes in the range $8-20 \mathrm{~Hz}$ concerned with body (P and S) waves. The form of amplitude spectrums of the "low-frequncy" events is shown in Figure $2 \mathrm{~b}$ using bold dashed line.

As was mentioned, the "low-frequency" waves are observed only on some of the registered events (about 5-10\% of overall quantity). Appearance of intensive Rayleigh waves on recordings is found to be not related with the effects of wave propagation paths. So there is a specific low-frequency physical process in the source of "low-frequency" events. Try to find out what sort it could be.

\section{PROBABLE SOURCE MECHANISMS OF THE "LOW. FREQUENCY" SEISMIC EVENTS}

The classical seismological source in the form of expanding shear crack and dynamical processes that take place in potash mines (Figure 3) have been examined as the potential sources of the "low-frequency" events.

Analysis for each process included the following stages:

- consideration of the mechanics of the process;

- construction of equivalent point source;

- calculation of synthetic seismograms;

- comparison of synthetic seismograms with waveforms of the "low-frequency" events.

The described analysis scheme implies the validity of the point source approximation. This requires that dominant wavelengths be greater than source dimensions and, at the same time, be lesser then distance to the point of observation. The dominant wavelengths in our case are $100-500 \mathrm{~m}$ (for body waves in the $8-20 \mathrm{~Hz}$ range) and $500-2000 \mathrm{~m}$ (for Rayleigh waves within the $0.5-2 \mathrm{~Hz}$ range). The processes under consideration have source sizes lesser than $100 \mathrm{~m}$. So on distances greater than $2000 \mathrm{~m}$ the point source approximation can be used. a

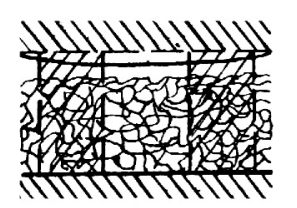

b

C

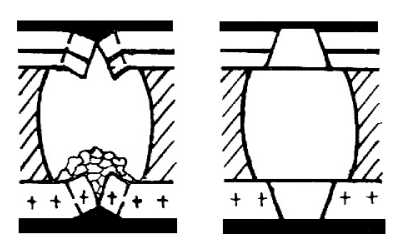

FIG. 3 Dynamical processes observed in potash mines: pillar burst (a), rockfall (b) and dynamical fracture of roof and floor (c) (modified after Dolgov et al., 1987)

\subsection{Shear Failure}

Elastic waves radiated by shear failure are described satisfactorily by a point source in the form of pair of force couples without a total torque (double couple). Amplitudes and directions of the force dipoles depend on the shape of fault surface and the displacement discontinuity distribution. In our study we have used the widespread faulting model of Sato and Hirasawa in the form of planar circular crack (Aki and Richards, 1980). Synthetic seismograms calculations for equivalent point sources obtained on the basis of Sato and Hirasawa's model were carried out by means of discretewavenumber integration method (Bouchon, 1981; Kennet and Kerry, 1979). Medium model in the form of layered half-space has been built on the basis of geological and geophysical data. Point source (double couple) was placed at a depth of $280 \mathrm{~m}$ (above mine openings level). Vertical components of particle velocities in points at the depth of $300 \mathrm{~m}$ 
(on a mine openings level) and at the different distances from the source $(2000 \mathrm{~m}, 3000 \mathrm{~m}, 5000 \mathrm{~m})$ were calculated. Synthetic waveforms obtained for one of the source variant ( $5 \mathrm{~m}$ radius vertical crack, rupture velocity $-2500 \mathrm{~m} / \mathrm{s}$, shear stress drop $-1 \mathrm{MPa}$ ) are represented in Figure 4a. Amplitude spectrums of these waveforms are shown in Figure $4 \mathrm{~b}$.

As it is seen, body waves are dominated on the waveforms. Amplitudes of surface waves are small. They are observed only in frequency domain (in the range 1-2 Hz). Similar features of synthetic waveforms remain valid under broad variation of parameters of the source model - fault plane orientation, radius (up to $10 \mathrm{~m}$ ) and rupture velocity (from 100 to $2500 \mathrm{~m} / \mathrm{s}$ ). Thus classical model of the source in the form of expanding shear crack does not reproduce waveforms that are typical for the "low-frequency" events.

As is obvious, the effect of the presence of mine openings in the vicinity of the shear failure has not been taken into account in the construction of equivalent point source. Consideration of this effect, as was shown in (Malovichko, 2004), results in addition of a complementary point source to a conventional double couple. This complementary source calculation shows to have a smaller magnitude than a primary source (double couple), on the one hand, and to radiate high-frequency seismic waves, on the other hand. Thus taking into account the distortion of shear failure-generated seismic waves by mine openings does not modify sufficiently the features of the synthetic waveforms (Figure 4).

\subsection{Pillar Burst}

A pillar burst has been described as the "step-wise" change of the vertical component of the traction above and below the pillar. The construction of equivalent point sources was realized by means of the approach presented in (Malovichko, 2004). A vertical linear dipole is dominated in the obtained point source. Its amplitude depends on source process parameters: traction drop magnitude and geometrical characteristics of the pillar and mine openings. For instance a source time function of the dipole in the case of $2 \mathrm{~m}$ width $6 \mathrm{~m}$ height and $10 \mathrm{~m}$ length intermediate pillar and $10 \mathrm{MPa}$ traction drop has a "step-wise" form with amplitude $-2.5 \cdot 10^{9}$ $\mathrm{N} \cdot \mathrm{m}$. Seismogram synthesized for such example and its spectrums are represented in Figure 5. Rayleigh waves may be observed on the waveforms, but their amplitudes, as in the previous case, are lesser than the body waves amplitudes. This relation does not change under the variation of pillar burst model parameters. Hence seismic waveforms for pillar burst do not manifest the features of the "low-frequency" events recordings.

\subsection{Rockfall}

Equivalent point source in the form of vertical single force is obtained for a local rockfall (Figure 3b). The single force amplitude behavior (source function) in time domain has a descriptive mechanical interpretation. The negative impulse (upward impact) is noted initially in the source function. It corresponds to an elastic rebound of the ambient medium due to separation of the collapsing block. Amplitude of the negative impulse equals to the weight of the block and its duration equals to the free-fall time of the block. The subsequent positive impulse describes impact of the collapsed block into mine opening floor.

Amplitude and duration of positive impulse is defined by a character (elastic, inelastic) of the block and the floor interaction. Synthetic waveforms calculated for a single force corresponding to rockfall of 50 tons in a $6 \mathrm{~m}$ height opening with inelastic "block-floor" interaction are demonstrated in Figure 6a. It is seen that features of the "low-frequency" events recordings (amplitudes of surface waves, relation of amplitudes of surface and body waves) are reproduced. In the case of elastic "block-floor" interaction synthetic waveforms become high-frequency and resemblance to the "low-frequency" recordings disappears.

\subsection{Floor Failure}

Dynamical failure of salt beds in a floor of a mining opening has been analyzed. Equivalent point source, as in the case of a pillar burst, has been obtained in the form of vertical linear dipole with a "step-wise" time function. It is characteristic a

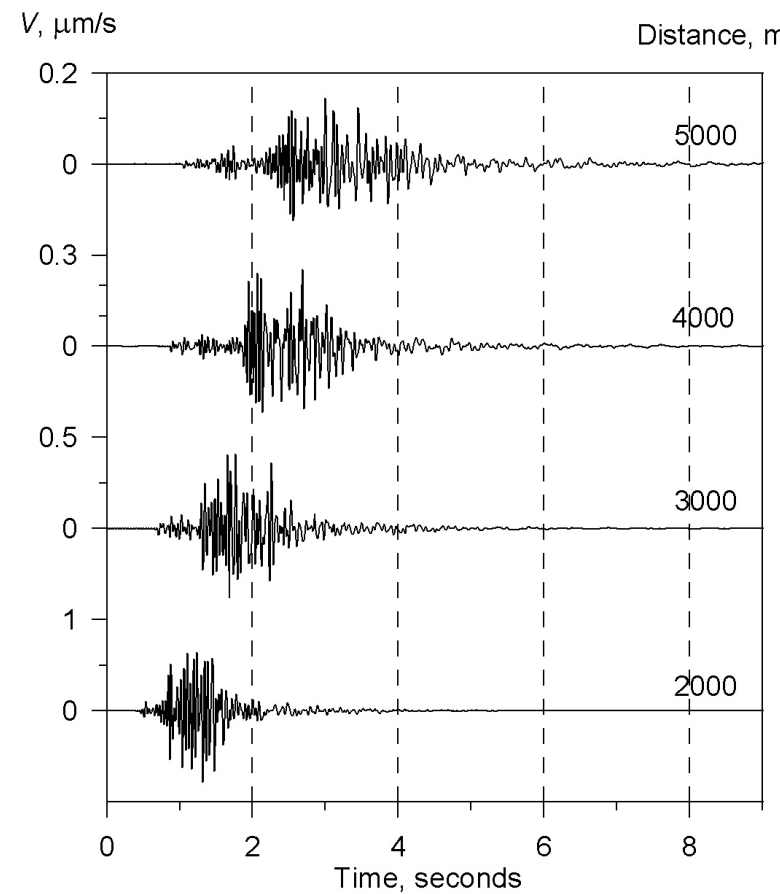

b

\section{$V,(\mu \mathrm{m} / \mathrm{s}) / \mathrm{Hz}$}

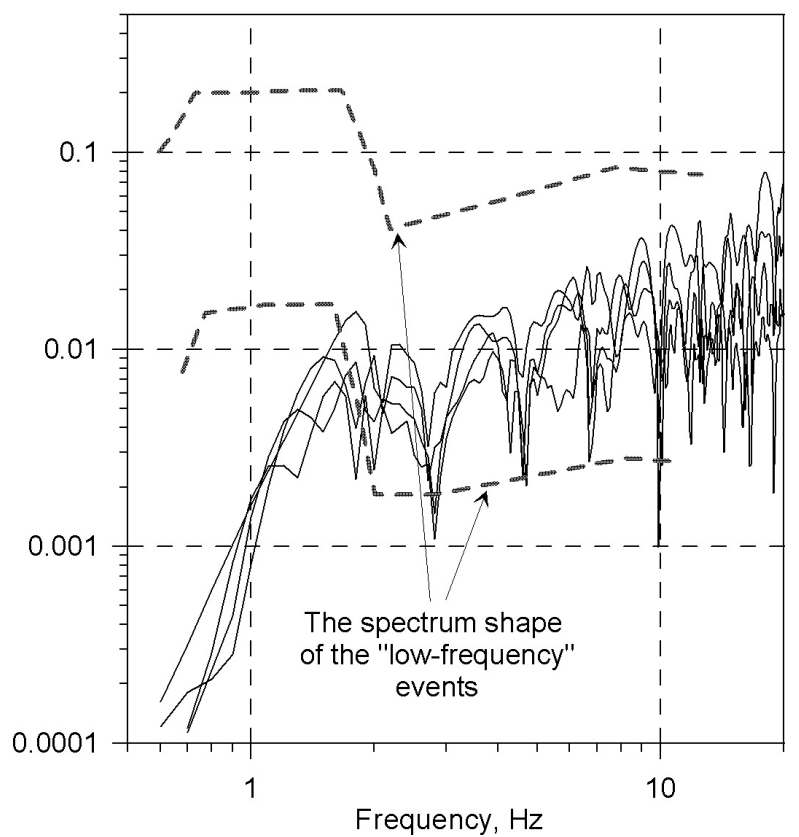


$V, \mu \mathrm{m} / \mathrm{s}$

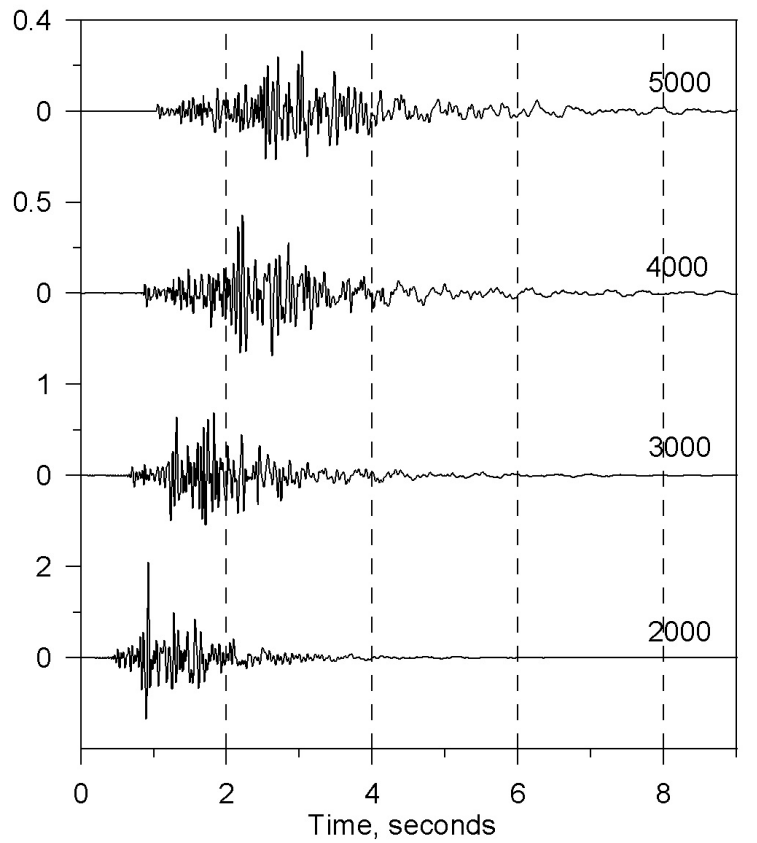

b

$V,(\mu \mathrm{m} / \mathrm{s}) / \mathrm{Hz}$

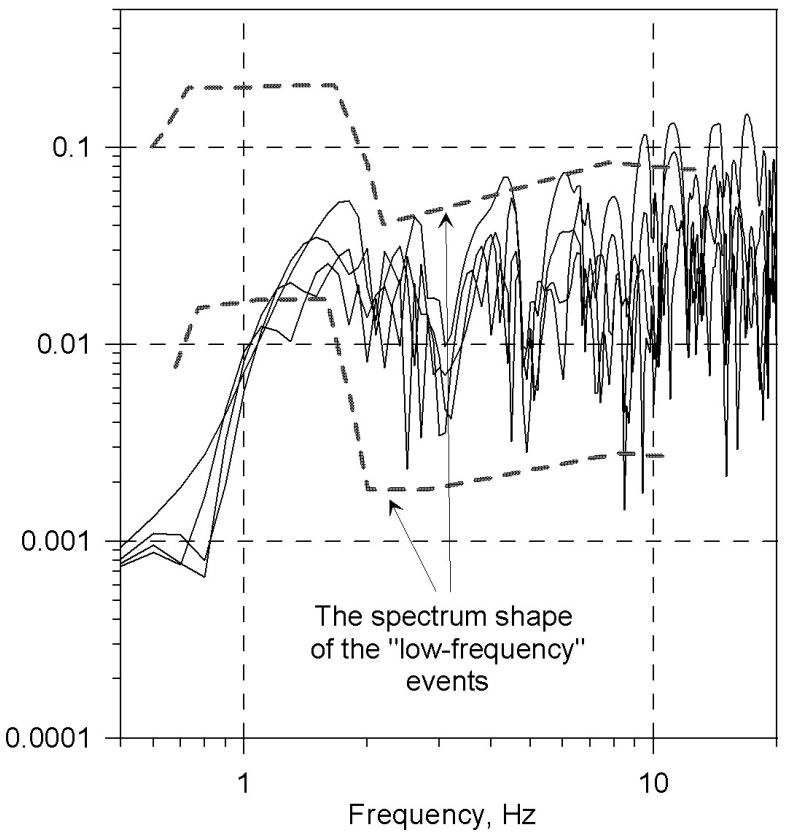

FIG. 5 Pillar burst synthetic waveforms (a) and their amplitude spectrums (b)

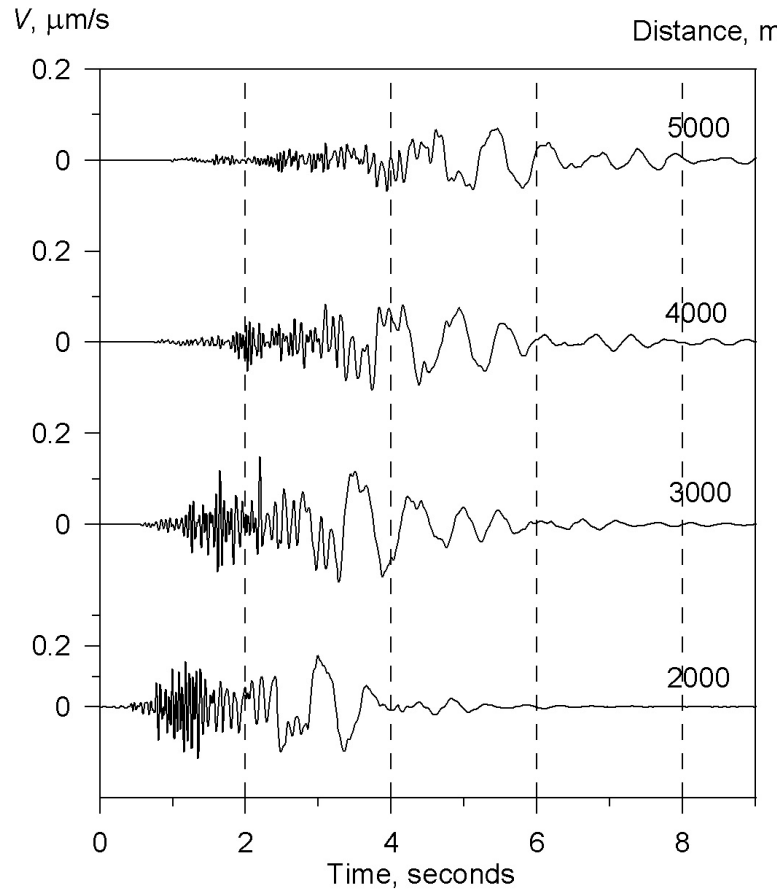

b

$V,(\mu \mathrm{m} / \mathrm{s}) / \mathrm{Hz}$

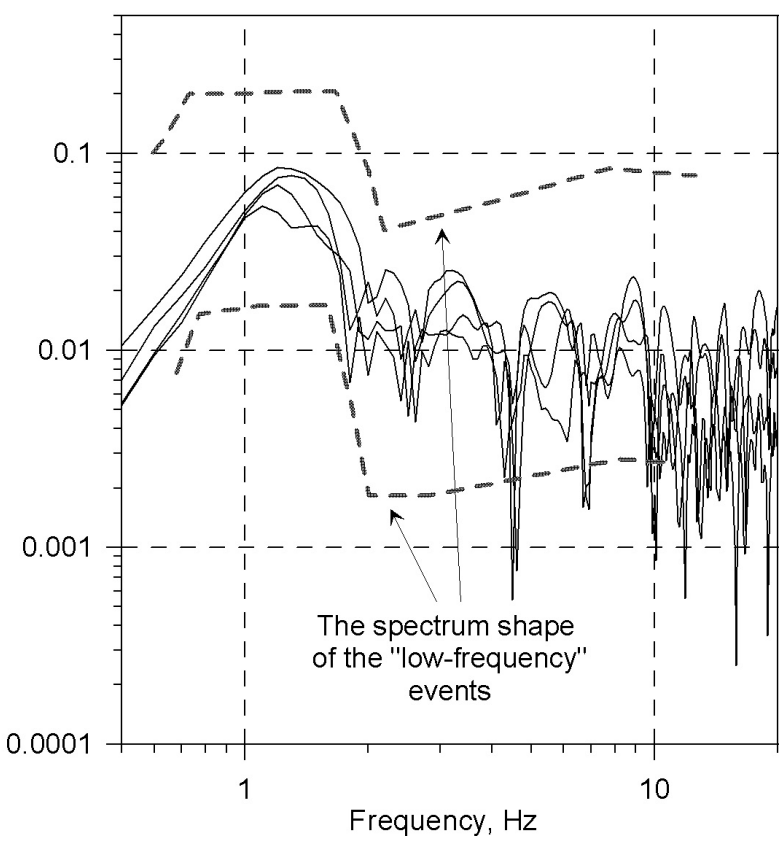

FIG. 6 Rockfall synthetic waveforms (a) and their amplitude spectrums (b) 


\section{$V, \mathrm{~nm} / \mathrm{s}$}

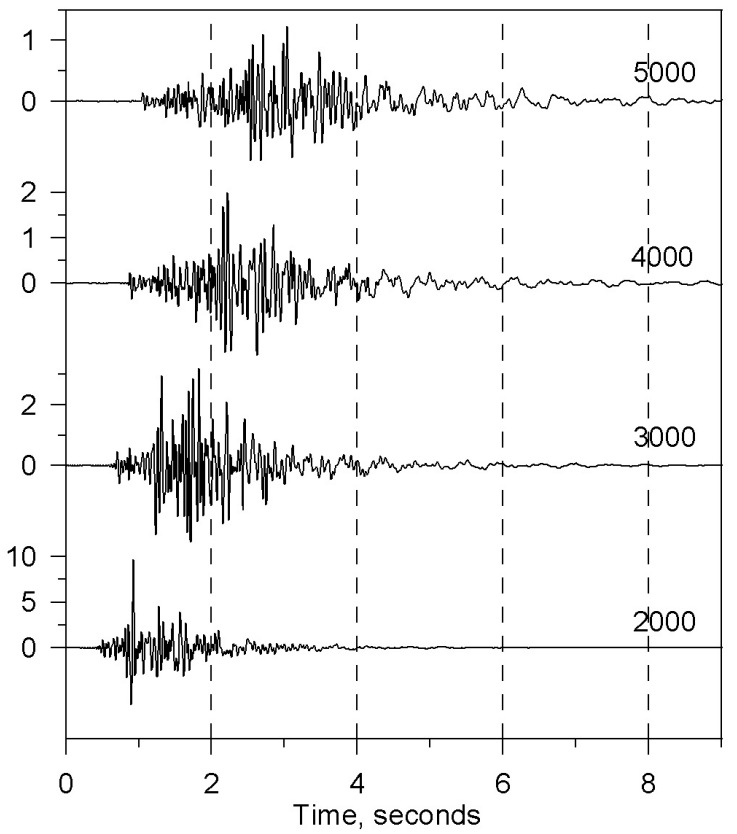

b

$V,(\mu \mathrm{m} / \mathrm{s}) / \mathrm{Hz}$

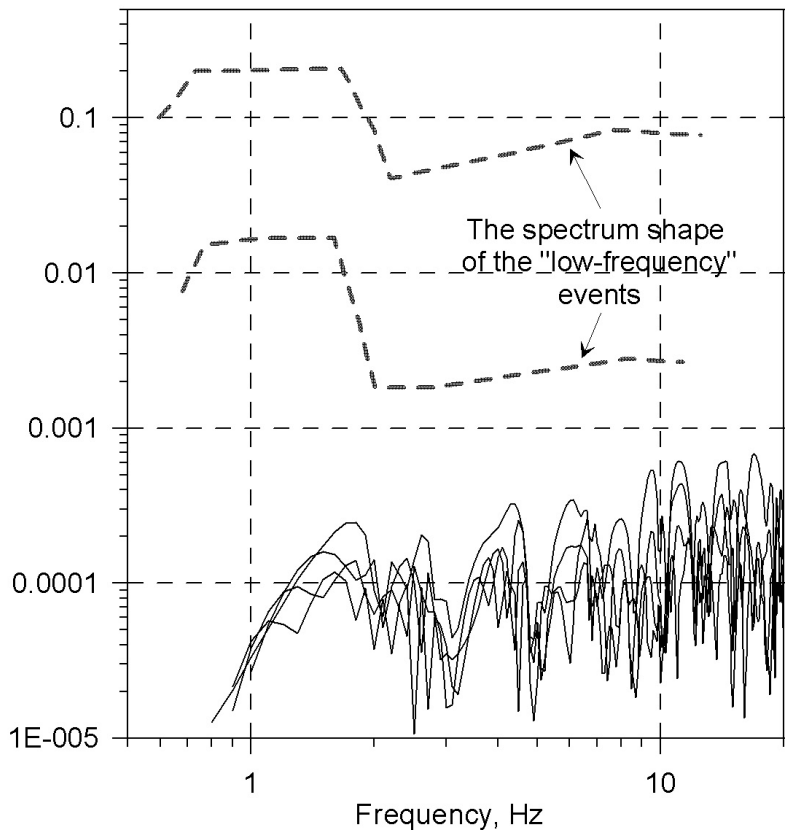

FIG. 7 Floor failure synthetic waveforms (a) and their amplitude spectrums (b)

that amplitude of the dipole for plausible process parameters (thickness and horizontal sizes of the salt bed, strength characteristics of the bed material) is sufficiently lower than in the case of the pillar burst. For instance, for the floor failure with maximum possible model parameters (salt bed sizes: height $1 \mathrm{~m}$, length $5 \mathrm{~m}$, width $3 \mathrm{~m}$; opening sizes: height $5 \mathrm{~m}$, width $5 \mathrm{~m}$; salt material tensile strength $1 \mathrm{MPa}$ ) amplitude of the equivalent vertical linear dipole is $8.7 \cdot 10^{6} \mathrm{~N} \cdot \mathrm{m}$. So floor failure has a small enough seismic efficiency. Corresponding synthetic seismogram is represented in Figure 7a. As it is seen from amplitude spectrums (Figure $7 \mathrm{~b}$ ), on the one hand, synthetic waveforms are high frequency enough and, on the other hand, amplitudes of the signals are lower than level of the "low-frequency" events recordings.

\section{CONCLUSIONS}

In this paper we have tried to find out what physical process could be responsible for appearance of the intensive lowfrequency Rayleigh waves on recordings of some events in the Verkhnekamskoye potash deposit. Seismic radiation of possible source processes has been analyzed by means of equivalent point source representation. Calculation of synthetic waveforms and their comparison with seismograms of the "low-frequency" events permits to conclude that a rockfall is the most probable source of the "low-frequency" seismic events.

The obtained result has qualitative interpretation. The lowering of the frequency content of the radiated seismic waves is defined by the following features of equivalent point source representation:

- presence of the low-degree polynomial moment of the equivalent forces, and

- presence of the low-frequency components in the source time function.

A rockfall fulfils both of these requirements. On the one hand, it has a non-zero polynomial moment of the equivalent forces with lowest degree, i.e. single force. On the other hand, its source time function contains low-frequency $(0.5-1 \mathrm{~Hz})$ component due to collapsed rocks free-fall time. The other considered processes (shear failure, pillar burst, floor failure) have more differentiated equivalent point source presentation (combinations of force dipoles).

\section{ACKNOWLEDGMENTS}

This work is supported by the Russian Foundation for Basic Research (grant N 04-05-65289). I would like to thank potash mining company JSC "Silvinit" (Solikamsk) which supported seismological investigation in the Verknekamskoye potash deposit over the years.

\section{REFERENCES}

Aki, K. and Richards, P.G. (1980) Quantitative seismology: theory and methods. W.H.Freeman, San Francisco, USA, 932 pages.

Bouchon, M. (1981) A simple method to calculate Green's function for elastic layered media. Bull. Seism. Soc. Am, v.71, pp. 959-971.

Dolgov, P.V., Polyanina, G.D. and Zemskov, A.N. (1987) Methods of prediction and prevention of gas-dynamic events in potash mines. Nauka, Alma-Ata, USSR, 176 pages (in Russian).

Kennet, B.L.N. and Kerry, N.J. (1979) Seismic waves in a stratified half space. Geophys. J. R. astr. Soc., v.57, pp. 557-583.

Malovichko, D.A. and Baranov, Yu.V. (2001) Application of numerical modelling for analysis of underground recordings of seismic events. In Proc. of the 5th Symposium on Rockbursts and seismicity in mines, Megaliesberg, 2001 (eds. G. van Aswegen, R.J.Durrheim, W.D.Ortlepp) SAIMM, Johannesburg, South Africa, pp. 479-482.

Malovichko, D.A. (2004) Equivalent point sources for seismogenic processes in mines. (unpublished).

Mining in the Verknekamskoye potash deposit methodical manual (1992) Ed. V.A.Soloviev, Nedra, Moscow, Russia, 468 pages (in Russian). 